Dokumentation des Wissenschaftlichen Symposiums

Verteilungsgerechtigkeit im Gesundheitswesen Probleme und Positionen am Beispiel der Transplantationsmedizin

7.-9. Mai 1992, Hannover

Veranstalter:

Akademie für Ethik in der Medizin, Göttingen

Akademie der Wissenschaften und der Literatur, Mainz

Deutsche Stiftung Organtransplantation, Neu-Isenburg

mit Unterstützung:

des Bundesministeriums für Gesundheit, sowie

des Niedersächsischen Ministeriums für Wissenschaft und Kultur 


\section{E. Nagel Ch. Fuchs (Hrsg.)}

\section{Soziale \\ Gerechtigkeit im \\ Gesundheitswesen}

Ökonomische, ethische, rechtliche Fragen am Beispiel der Transplantationsmedizin

Unter Mitarbeit von

M. Niechzial, R. Pichlmayr und P. Schölmerich

Mit 15 Abbildungen und 31 Tabellen

Springer-Verlag

Berlin Heidelberg New York 1993

London Paris Tokyo

Hong Kong Barcelona

Budapest 
Dr. med. Eckhard Nagel

Medizinische Hochschule Hannover

Klinik für Abdominal- und Transplantationschirurgie

Konstanty-Gutschow-Straße 8

30625 Hannover

Professor Dr. med. Christoph Fuchs

Bundesärztekammer Köln

Herbert-Lewin-Straße 1

50931 Köln

Die Abbildung auf dem Einband zeigt eine Darstellung der Justitia (glasierter Ton). (Aus Planiscig L (1940) Luca Della Robbia, Verlag Anton Schroll \& Co., Wien)

\section{ISBN 3-540-56892-1 Springer-Verlag Berlin Heidelberg NewYork}

Die Deutsche Bibliothek - CIP-Einheitsaufnahme. Soziale Gerechtigkeit im Gesundheitswesen : ökonomische, ethische, rechtliche Fragen am Beispiel der Transplantationsmedizin ; mit 31 Tabellen ; [Dokumentation des Wissenschaftlichen Symposiums Verteilungsgerechtigkeit im Gesundheitswesen Probleme und Positionen am Beispiel der Transplantationsmedizin, 7.-9. Mai 1992, Hannover] / E. Nagel ; CH. Fuchs (Hrsg.). Unter Mitarb. von M. Niechzial . . . [Veranst.: Akademie für Ethik in der Medizin, Göttingen ... . . - Berlin ; Heidelberg ; New York ; London ; Paris ; Tokyo ; Hong Kong ; Barcelona ; Budapest : Springer, 1993

ISBN 3-540-56892-1

Dieses Werk ist urheberrechtlich geschützt. Die dadurch begründeten Rechte, insbesondere die der Ubersetzung, des Nachdrucks, des Vortrags, der Entnahme von Abbildungen und Tabellen, der Funksendung, der Mikroverfilmung oder der Vervielfältigung auf anderen Wegen und der Speicherung in Datenverarbeitungsanlagen, bleiben, auch bei nur auszugsweiser Verwertung, vorbehalten. Eine Vervielfältigung dieses Werkes oder von Teilen dieses Werkes ist auch im Einzelfall nur in den Grenzen der gesetzlichen Bestimmungen des Urheberrechtsgesetzes der Bundesrepublik Deutschland vom 9. September 1965 in der jeweils geltenden Fassung zulässig. Sie ist grundsätzlich vergütungspflichtig. Zuwiderhandlungen unterliegen den Stafbestimmungen des Urheberr echtsgesetzes.

(C) Springer-Verlag Berlin Heidelberg 1993

Printed in Germany

Die Wiedergabe von Gebrauchsnamen, Handelsnamen, Warenbezeichungen usa. in diesem Werk berechtigt auch ohne besondere Kennzeichnung nicht zu der Annahme, daß solche Namen im Sinne der Warenzeichen- und Markenschutz-Gesetzgebung als frei zu betrachten wären und von jedermann benutzt werden dürften.

Satz: Reproduktionsreife Vorlage vom Autor

24/3130-543210-Gedruckt auf säurefreiem Papier

Bayerische

Staatsbibliothek

München 


\section{Inhaltsverzeichnis}

Einführung durch die Veranstalter $\ldots \ldots \ldots \ldots \ldots \ldots$ XVII

Allokationswirklichkeit unseres Gesundheitswesens $\ldots \ldots \ldots 1$

Einführung

R. Grupp . . . . . . . . . . . . . . . . 3

Allokationsprobleme bei knappen Ressourcen

Ch. Fuchs . . . . . . . . . . . . . . . . . . . 6

Entscheidungsprozesse im Gesundheitswesen

F. W. Schwartz . . . . . . . . . . . . . . . . . . 21

Beitragsstabilität und Allokationswirklichkeit

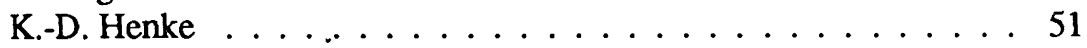

State of the art: Möglichkeiten in der Nieren-, Leber-

und Knochenmarktransplantation . . . . . . . . . . . . . 79

Einführung

E. Nagel, H.-W. Schreiber . . . . . . . . . . . . . 80

Möglichkeiten in der Nieren- und Lebertransplantation

R. Pichlmayr . . . . . . . . . . . . . . . 82

Möglichkeiten und Grenzen der Knochenmarktransplantation

H. Link . . . . . . . . . . . . . . . . . . . . . . . . . . . . . 97

Indikation und Bedarf bei der Nieren-, Leber-

und Knochenmarktransplantation . . . . . . . . . . . . . . . 109

Einführung

P. Schölmerich . . . . . . . . . . . . . . . . . . . 110

Indikation und Bedarf

H. Raspe . . . . . . . . . . . . . . . . . . 111

Indikation und Bedarf bei der Lebertransplantation

M.P. Manns, K. Böker . . . . . . . . . . . . . . . . . . . . . . . . 129

Indikation und Bedarf bei der Nierentransplantation

W. Schoeppe . . . . . . . . . . . . . . . . . . . . 143

Indikation und Bedarf bei der Knochenmarktransplantation

H. Riehm . . . . . . . . . . . . . . . . . . 151 
Struktur, Organisation und ökonomische Grundlagen

der medizinischen Versorgung im Hinblick

auf die Allokationswirklichkeit . . . . . . . . . . . . . . . 157

Ökonomische Grundlagen der Krankenhausfinanzierung

K.-H. Tuschen . . . . . . . . . . . . . . . . . . 158

Kosten-Nutzen-Analysen im Gesundheitswesen

J.-M. Graf v. d. Schulenburg, 0. Schöffski . . . . . . . . . . . 168

Voraussetzungen für Nieren- und Lebertransplantationen

E. Nagel . . . . . . . . . . . . . . . . . . . . . . . 186

Voraussetzungen für die Knochenmarktransplantation

H. Link . . . . . . . . . . . . . . . . . . . . . . 200

Medizinische Verteilungsentscheidungen

und ihre Rechtfertigung . . . . . . . . . . . . . . . . . . . 209

Einführung

G. Patzig . . . . . . . . . . . . . . . . . . . 210

Lebensqualität - Entscheidungshilfen durch ein neues

evaluatives Konzept?

M. Bullinger . . . . . . . . . . . . . . . . . . . . . 214

Entscheidungskriterien und Konflikte am Beispiel

der Nierentransplantation

E. Renner

Entscheidungskriterien und Konflikte am Beispiel

der Lebertransplantation

F.W. Eigler . . . . . . . . . . . . . . . . . . . . . . 242

Philosophische Aspekte der Güterverteilung . . . . . . . . . . . . 249

Verteilungsgerechtigkeit aus philosophischer Sicht

J. Nida-Rümelin . . . . . . . . . . . . . . . . . . . . . . 250

"Gerechtigkeitskriterien" in der Transplantationsmedizin -

Eine ordoliberale Perspektive

H. Kliemt . . . . . . . . . . . . . . . . . . . 262

Verteilungsgerechtigkeit aus juristischer Sicht . . . . . . 285

Einführung

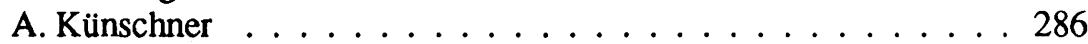


Standards, Kostendruck und Haftpflichtrecht

A. Laufs . . . . . . . . . . . . . . . . . . . . . . . . . . 290

Rechtliche Kriterien der Verteilungsgerechtigkeit

im Sozialstaat

H.-L. Schreiber . . . . . . . . . . . . . . . . . . 302

Verteilungsgerechtigkeit im Gesundheitswesen -

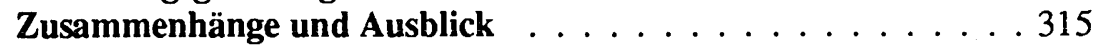

Zusammenhänge für die Gesundheitspolitik

R. Grupp . . . . . . . . . . . . . . . . 316

Zusammenhänge aus der Sicht der Krankenkassen

F. J. Oldiges . . . . . . . . . . . . . . . . . . . . . 324

Abschlußdebatte . . . . . . . . . . . . . . 332

Sachverzeichnis . . . . . . . . . . . . . . . 343 


\section{Verzeichnis der Autoren und Referenten}

K. Böker, Dr. med.

Abt. für Gastroenterologie

und Hepatologie

Medizinische Hochschule Hannover

30625 Hannover

M. Bullinger, PD Dr. phil.

Institut für Medizinische Psychologie

Goethestraße 31

80336 München

F.-W. Eigler, Prof. Dr. med.

Abt. für Allgemeine Chirurgie

Universitätsklinikum Essen

Hufelandstraße 55

43147 Essen

J. M. Graf v. d. Schulenburg, Prof. Dr. Universität Hannover

Institut für Versichenungsbetriebslehre am Fachbereich Wirtschaftswisserisch. Wunstorfer Straße 14

30453 Hannover

R. Grupp, Ministerialdirigent, Dr.

Bundesministerium für Gesundheit

Rochusstraße 1

53123 Bonn

K.-D. Henke, Prof. Dr.

Abt. für öffentliche Finanzen am

Fachbereich Wirtschaftswissenschaften

Universität Hannover

Wunstorfer Straße 14

30453 Hannover

H. Kliemt, Prof. Dr. phil.

Gesamthochschule Duisburg

Fachbereich I Philosophie

4100 Duisburg

A. Künschner, Dr. jur., LL.M.

Kronenstraße 18

79100 Freiburg/Breisgau
A. Laufs, Prof. Dr. jur., Dr. h.c.

Rupprecht-Karls-Universität

Institut für Geschichtliche

Rechtswissenschaft

Friedrich-Ebert-Platz 2

69117 Heidelberg

H. Link, PD Dr. med.

Klinik für Hämatologie und Onkologie

Medizinische Hochschule Hannover

30625 Hannover

M. Manns, Prof. Dr. med.

Abt. f. Gastroenterologie u. Hepatologie Medizinische Hochschule Hannover 30625 Hannover

J. Nida-Rümelin, Prof. Dr.

Eberhard-Karls-Universität

Zentrum für Ethik in den Wissenschaften

Wilhelmstraße 20

72074 Tübingen

M. Niechzial, Dr. med.

Medizinische Hochschule Hannover

Klinik für Abdominal- und

Transplantationschirurgie

Konstanty-Gutschow-Straße 8

30625 Hannover

F. J. Oldiges, Dr.

AOK-Bundesverband, Geschäftsfuihrung

Postfach 200844

53177 Bonn

G. Patzig, Prof. Dr.

Georg-August-Universität

Philosophisches Seminar

Platz der Göttinger Sieben 5

37073 Göttingen

R. Pichlmayr, Prof. Dr. med.

Klinik für Abdominal- und

Transplantationschinurgie

Medizinische Hochschule Hannover

30625 Hannover 
H. Raspe, Prof. Dr. med., Dr. phil. Medizinische Universität zu Lübeck Institut für Solarmedizin

Sophienstraße 2

23560 Lübeck

E. Renner, Prof. Dr. med.

Städtisches Krankenhaus Köln-Merheim Medizinische Klinik I

Ostmerheimer Straße 200

51109 Köln

H. Riehm, Prof. Dr. med.

Abt. für Päd. Hämatologie u. Onkologie Kinderklinik der

Medizinischen Hochschule Hannover

30625 Hannover

O. Schöffski, Dipl.-Öko.

Universität Hannover

Institut für Versicherungsbetriebslehre am Fachbereich Wirtschaftswissensch.

Wunstorfer Straße 14

30453 Hannover

W. Schoeppe, Prof. Dr. med.

Johann Wolfgang v. Goethe-Universität

Zentrum für Innere Medizin

Theodor-Stern-Kai 7

60596 Frankfurt
P. Schölmerich, Prof. Dr. med., Dr. h.c. em. Direktor der Medizinischen

Universitätsklinik Mainz

Weidmannstraße 67

55131 Mainz

H.-L. Schreiber, Prof. Dr. jur., Dr. h.c.

Georg-August-Universität

Juristisches Seminar

Platz der Göttinger Sieben 6

37073 Göttingen

H.-W. Schreiber, Prof. Dr. med.

em. Direktor der Abt. für Allgemeine

Chirurgie der Universitätsklinik

Eppendorf

Martinistraße 52

20251 Hamburg

F. W. Schwartz, Prof. Dr. med.

Abt. für Epidemiologie und

Sozialmedizin

Medizinische Hochschule Hannover

30625 Hannover

K.-H. Tuschen

Regierungsdirektor

Bundesministerium für Gesundheit

Rochusstraße 1

53123 Bonn 


\title{
Verteilungsgerechtigkeit aus philosophischer Sicht
}

\author{
J. Nida-Rümelin*
}

\section{Utilitaristisch-konsequentialistische Rationalität}

Wenn man einen weiten Gesundheitsbegriff zugrunde legt, wie ihn etwa die WHO 1948 im Sinne eines physischen, mentalen und sozialen Wohlbefindens definiert hat, wenn man zum zweiten an einer Overall-Betrachtung - manche Philosophen nennen dies eine universal-teleologische Betrachtung - festhält, d. h. wenn man sich überlegt, in welcher Weise die Medizin, gesamtgesellschaftlich betrachtet, dazu beitragen kann, Gesundheit zu optimieren, liegt es nahe, die Medizinethik unter die Kriterien utilitaristischkonsequentialistischer Rationalität zu stellen; utilitaristisch deshalb, weil das zu optimierende Gut das subjektive Wohlbefinden ist, und konsequentialistisch insofern, als das, was in der medizinischen Praxis geschieht, instrumentell auf die Optimierung dieses Gutes ausgerichtet ist.

\section{Grenzen kosequentialistischer Medizinethik}

(1) Weiterer

Gesundheitsbegriff

(2) "Overall"-Betrachtung (universal-teleologisch)
Medizinethik unter

Kriterien utilitaristischkonsequen-tialistischer Rationalität

Einwände:

(a) Paternalismus

(b) überbordende Verantwortlichkeit

(c) Gerechtigkeitsindifferenz

(d) Verletzung von Individualrechten

(e) Kooperationsproblematik

* Zentrum für Ethik in den Wissenschaften der Eberhard-Karls-Universität, Tübingen 
Es gibt eine ganze Reihe sehr grundsätzlicher Einwände gegen konsequentialistische Kriterien in der Ethik und in der Theorie praktischer Rationalität generell. Für dieses Symposium ist interessant, daß gerade auch Erfahrungen in der Diskussion medizinethischer Probleme - vor allem im angelsächsischen Sprachraum - dazu geführt haben, daß dieses im angelsächsischen Sprachraum dominierende utilitaristische Paradigma in der Ethik zunehmend in Frage gestellt wurde.

\section{Einwände}

Ich will hier nur vier grundlegende Einwände stichwortartig nennen.

1. Es handelt sich zum einen um den Einwand des Paternalismus. Wenn jemand auf Grund seiner spezifischen Kenntnisse und Erfahrungen - etwa ein Arzt - zu wissen glaubt, was - im Sinne der Optimierung von Gesundheit - gut ist für einen Patienten, dann wäre es ihm aufgetragen, den Patienten zu veranlassen, dementsprechend zu handeln. Wenn der Patient Widerstand zeigt, muß der Arzt dies notfalls mit Überredung zu verändern suchen. Dagegen stehen Intuitionen, die man aus dem Alltagsleben und sicher auch aus der medizinischen Praxis hat, die besagen: Jeder muß für sich selber wissen, was für ihn gut ist, die letzte Entscheidung liegt bei dem entsprechenden Individuum; auch dann, wenn ich hundertprozentig davon überzeugt bin, daß etwas für ihn nicht gut ist, bin ich nicht berechtigt, diese Widerstände in irgendeiner Form zu überwinden, auch dann nicht, wenn dem beispielsweise keine rechtlichen Probleme entgegenstünden.

2. Der zweite Einwand lautet: Wenn man von der einzelnen Handlung verlangt, daß sie sich an diesem Optimierungsmodell orientiert, dann hat das zur Folge, was ich einmal als "überbordende Verantwortlichkeit" bezeichnet habe, d. h. die einzelne Entscheidung muß jeweils alle möglichen Handlungsoptionen in diesem Zeitpunkt berücksichtigen und die dann optimale Handlung auswählen. Das bedeutet, daß Projekte nicht mehr verfolgt werden können, weil man bei diesem Abwägungsprozeß permanent von dem abweichen muß, was man ursprünglich vorhatte. Eine weitere Folge ist, daß die persönliche Integrität der handelnden Person gefährdet ist. Ich glaube, das gilt wiederum in besonderem Maße für die medizinethische Fragestellung.

3. Der dritte Einwand lautet, daß, wenn es ausschließlich um die Frage geht, wie ein bestimmtes Gut zu optimieren sei, Gerechtigkeits- und Verteilungsaspekte im eigentlichen Sinne vernachlässigt werden. Auf diesem Symposion wurde bereits darüber diskutiert, ob die Histokompatibilität das ausschließliche Kriterium sein muß. Ein Argument in dieser Richtung lautet: Ressourcen sind knapp, also müssen sie effizient eingesetzt werden. Effizienz könnte man übersetzen mit: qualifizierte Erhöhung der Lebenserwartung. Wenn man das in jedem Einzelfall tut, nimmt man implizit für ein ethisches Kriterium Stellung, das Verteilungsaspekten gegenüber indifferent ist. Es geht um die Optimierung der Gesundheit sozusagen in der 
Summe, in der Bilanz der Verlängerung der Lebenserwartung in der Gesellschaft insgesamt. Verteilungsfragen bleiben außen vor, und das ist auch wünschenswert, nämlich unter dem Gesichtspunkt der Verantwortungsentlastung.

4. Ein weiterer Einwand befaßt sich mit der Verletzung von Individualrechten. Beispielsweise ist selbstverständlich, daß es nicht zulässig ist - und zwar nicht nur juristisch, sondern auch ethisch -, das Leben eines potentiellen Spenders zu verkürzen, um in der Summe möglicherweise eine optimale Lebenszeitverlängerung im Erwartungswert zu erreichen. Wenn man nur diese Aggregationsvorstellung hat, wenn man nur die Lebenserwartungsverlängerung aufsummiert, dann ist dieses moralische Verbot nicht legitimierbar, jedenfalls nicht in direkter Form.

\section{Andere Kriterien der Verteilungsgerechtigkeit}

Nun sind in der Philosophie und in der Ökonomie eine ganze Reihe von Kriterien der Verteilungsgerechtigkeit in der Diskussion. Ich will dabei zwei Hauptgruppen unterscheiden, und zwar zum einen diejenigen Kriterien von Verteilungsgerechtigkeit, die versuchen, auf die Problematik der interpersonellen Vergleichbarkeit zu verzichten. Man empfindet ein Unbehagen dabei, wenn man beispielsweise die Lebensqualitäten unterschiedlicher Personen miteinander vergleichen soll. In der Ökonomie war das lange Zeit geradezu ein Dogma - Stichwort: Ordinalismus: Es darf keine interpersonellen Vergleiche von Nutzenniveaus oder von Nutzeneinheiten geben.

\section{Pareto-Vergleichbarkeit}

Es gibt ein interessantes Theorem des indischen Ökonomen und Philosophen Amartya Sen, das folgenden logischen Zusammenhang feststellt: Wenn man ein Kriterium der Verteilungsgerechtigkeit entwickelt und dabei die folgenden fünf Bedingungen verlangt:

1. daß die Rangordnung der Verteilung unter diesem Gerechtigkeitskriterium ausschließlich eine Funktion der individuellen Präferenz der Betroffenen sein soll,

2. da $\beta$ dieses Kriterium vollständig ist, $d . h$. daß unter diesem Kriterium beliebige Verteilungen miteinander verglichen werden können,

3. daß die Stellung zweier Verteilungen in der Gerechtigkeitsrangordnung ausschließlich von den individuellen Präferenzen bezüglich dieser beiden Verteilungen oder Zustände abhängt,

4. daß, wenn mindestens eine Person eine strikte Präferenz für die eine Verteilung hat und keine Person eine umgekehrte Präferenz, diese Verteilung in der Gerechtigkeitsrangordnung höher steht,

5. daß Anonymität besteht, $d$. h. die Präferenzen als solche ändern sich nicht deshalb, weil andere Personen diese Präferenzen einnehmen,dann läßt sich zeigen, daß es nur noch ein Kriterium der Gerechtigkeit gibt, das mit diesen fünf Bedingungen kompatibel ist, nämlich: Eine Vertei- 
lung ist gerechter als eine andere, wenn sie Pareto-besser ist; Verteilungen, die nicht Pareto-vergleichbar sind, sind unter Gerechtigkeitsaspekten indifferent.

Pareto-besser bedeutet: Eine Verteilung ist dann besser, wenn es mindestens eine Person gibt, die sie vorzieht, und wenn es keine Person gibt, die die andere Verteilung vorzieht. Nicht Pareto-vergleichbar heißt, da $B$ diese Relation weder in der einen noch in der anderen Richtung besteht. Dies ist ein sehr attraktives Kriterium des Vergleichs von Allokationen, weil dadurch interpersonelle Vergleiche nicht erforderlich sind.

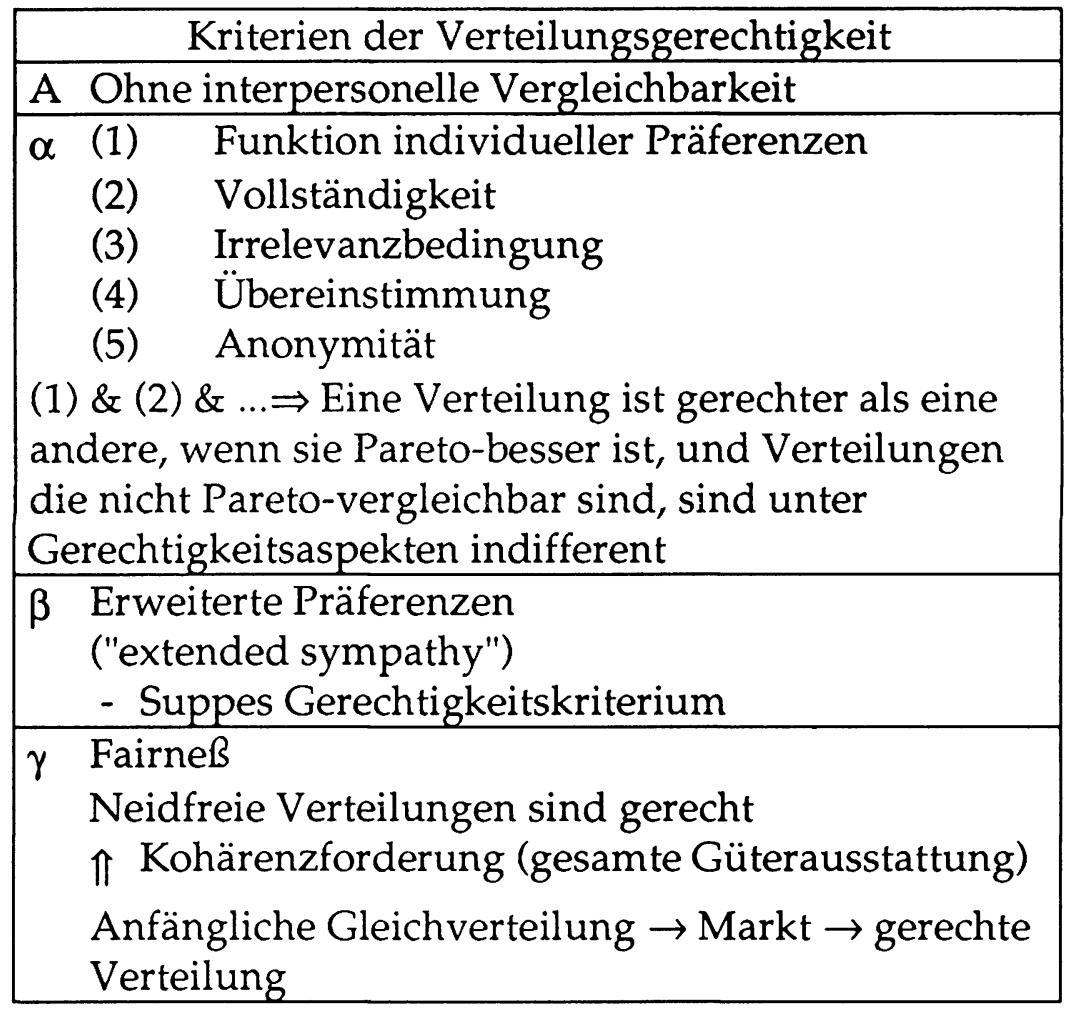

Dennoch ist klar: Gerade bei der Allokationsfrage im Hinblick auf die Transplantationsmedizin ist dieses Kriterium wenig hilfreich; denn es ist nicht anzunehmen, da $\beta$ es im Vergleich zueinander sehr viele Verteilungen gibt, die diese Bedingungen erfüllen. Es gibt einen offensichtlichen Interessenkonflikt einzelner Individuen zueinander. Das heißt, bei der Allokation einer begrenzten Anzahl von Spenderorganen auf eine größere Anzahl von potentiellen Empfängern ist jede Allokation mit den anderen Allokationen nicht Pareto-verglcichbar, und dieses Kriterium ist überhaupt keine Hilfe. 
Um dieses Problem zu umgehen, wurde der Ansatz erweitert, aber immer noch ohne interpersonelle Vergleichbarkeiten vorauszusetzen. Der Grundgedanke ist, da $\beta$ wir nicht nur einfach Präferenzen haben, sondern $\mathrm{da} B$ wir alle auch eine Vorstellung darüber haben, wie es anderen Personen in ihrem Zustand geht. Wir versetzen uns also in die Lage der anderen Personen und beurteilen dann: Wie würde ich meine Präferenzen zwischen den verschiedenen Verteilungen oder Zuständen gestalten? Wenn man das zugrunde legt und Pareto hinzunimmt, kommt man zu einem sehr viel vollständigeren Gerechtigkeitskriterium, nämlich dem Gerechtigkeitskriterium von Suppes.

\section{Neidfreie Verteilungen}

Es gibt eine weitere interessante Methode, Gerechtigkeitsfragen zu klären, ohne interpersonelle Vergleiche anzustellen. Der Grundgedanke lautet: Neidfreie Verteilungen, also Verteilungen, bei denen niemand auf die Position einer anderen Person neidisch ist, sind gerecht. Man könnte sagen: Das ist trivial und bringt überhaupt nichts, da die Menschen immer neidisch sind. Ganz so einfach kann man es aber nicht abtun, denn in diesem Konzept steckt eine strenge Kohärenzforderung. Es wird nicht die Neidfreiheit in bezug auf die Verteilung eines einzelnen Gutes verlangt, sondern in bezug auf die gesamte Güterausstattung der Personen. Das führt zu höchst interessanten Ergebnissen, wenn man beispielsweise unterschiedliche Produktivitäten, unterschiedliche Geschmäcker, unterschiedliche Fähigkeiten, unterschiedliche Mußebedürfnisse usw. zugrunde legt. Insbesondere läßt sich zeigen, daß sich bei einer anfänglichen Gleichverteilung, die einem kompetitiven Marktsystem ausgesetzt ist, eine gerechte Verteilung im Sinne dieser Neidfreiheit ergibt.

Man kann eine Ausdehnung auf Vergleiche zwischen Gruppen vornehmen und so einen Bezug zu diesem Symposium herstellen. Man vergleiche einmal die gesunden Beitragszahler und die Gruppe der potentiellen Transplantationspatienten. Es ist anzunehmen, da $\beta$ in der Regel gilt: Jede Person in der Gruppe der gesunden Beitragszahler möchte nicht tauschen mit der Güterausstattung der potentiellen Transplantationspatienten, und zwar unter den heutigen Bedingungen, bei den heutigen Beitragssätzen. Bis eine Person zu einem solchen Tausch bereit wäre, müßten die Beiträge der gesunden Personen extrem steigen. Wenn man dieses Konzept von Gerechtigkeit zugrunde legt, sind sicher die volkswirtschaftlichen Summen, die aufgewendet werden, noch viel zu niedrig, um Neidfreiheit herzustellen. Ganz anders sieht es natürlich aus, wenn man unterschiedliche Patientengruppen miteinander vergleicht. Herr Henke hat die Liste vorgestellt, nach der sich zeigen läßt, daß sich beispielsweise eine monetäre Ressource im Sinne von Lebensqualitätsverbesserung sehr viel effektiver bei anderen medizinischen Dienstleistungen einsetzen ließe. Dann entsteht natürlich sofort der umgekehrte Effekt, allerdings innerhalb der Patientenschaft insgesamt, nicht zwischen den Patienten und den Gesunden. 
Gerade deshalb, weil der Vergleich zwischen den Patienten ganz offensichtlich Schwierigkeiten bereitet, bietet es sich an, doch - wenn auch mit Bauchschmerzen - diese theoretische Abstinenz, also die Beschränkung auf Gerechtigkeitskriterien ohne interpersonelle Vergleichbarkeit, aufzugeben und in der einen oder anderen Weise - Frau Bullinger hat das methodische Instrumentarium skizziert - Vergleichsmaßstäbe einzuführen und sich zu überlegen: Angenommen, es gäbe einen Maßstab für Lebensqualität, welches Kriterium von Gerechtigkeit könnte man dann akzeptieren?

\section{Klasssischer Handlungsuilitarismus}

Ein Kriterium, das bei vielen Personen eine hohe Attraktivität besitzt, ist das Kriterium des klassischen Handlungsutilitarismus. Man sagt: Wenn ich schon ein solches Maß - das der Lebensqualität - habe, dann sollte die Summe der Lebensqualitäten über das Zeitintegral des Lebens maximiert werden. Das ist allerdings nicht so plausibel, wie es zunächst einmal erscheint.

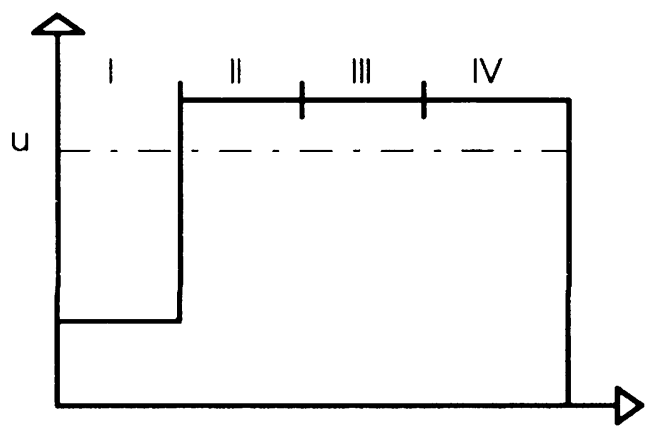

Nehmen wir an, es gäbe vier Gruppen von Patienten. Die dargestellten Flächen repräsentieren die Summe der Lebensqualität der Gruppen. Angenommen, man könnte durch eine bestimmte Allokation von der Gleichverteilung der Lebensqualität zu einer neuen Verteilung, der aufgezeigten Stufenverteilung, übergehen und diese wäre im Sinne der utilitaristischen Optimierung optimal (die jeweiligen Gesamtflächen sind nämlich fast gleich groß). Intuitiv spricht vieles dagegen, in der einen Gruppe die Lebensqualität extrem abzusenken, um anderen Patientengruppen eine etwas höhere Lebensqualität zu verschaffen.

\section{Ethischer Bayesianismus}

Eine weitere Variante, welche die interpersonelle Vergleichbarkeit nutzt, allerdings in weit geringerem Maße als der klassische Handlungsutilitarismus, bezeichnet man als ethischen Bayesianismus. Das ist insofern ganz attraktiv, als darauf verzichtet wird, Maße beispielsweise für Lebensqualität oder Nutzen einzuführen. Man beschränkt sich einfach darauf, wie das 
Entscheidungsverhalten von Personen ist. Vorausgesetzt, dies hat eine gewisse Kohärenz - eine idealisierende Annahme -, dann läßt sich zeigen, daß man je individuell eine (bis auf lineare Transformation eindeutig bestimmte) Nutzenfunktion zuordnen kann. Es wird ein Skalierungsverfahren vorgeschlagen, mit dem die im Präferenzverhalten der Personen zum Ausdruck kommenden subjektiven Bewertungen vergleichbar werden. Dieses wird dann optimiert. Diese Variante weist, was die Verteilungsgerechtigkeit angeht, dieselbe Problematik wie die erste Variante auf.

\begin{tabular}{|ll|}
\hline & Kriterien der Verteilungsgerechtigkeit \\
\hline $\boldsymbol{B}$ & Mit interpersoneller Vergleichbarkeit \\
\hline$\alpha$ & Handlungs-konsequentialistisch \\
& Klassischer Handlungsutilitarismus \\
& - Vergleichbarkeitsproblem \\
& - Medizinisches Berufsethos \\
& - Verteilungsindifferenz \\
\hline$\beta$ & Ethischer Bayesianismus \\
& - kein Vergleichbarkeitsproblem \\
& - sonst gleiche Problematik \\
\hline
\end{tabular}

Wir haben bisher nur von Verteilungen gesprochen, beispielsweise bei Allokationsentscheidungen. Aber nicht nur diese sind Gegenstand von Gerechtigkeitsgesichtspunkten. Es gibt Auffassungen, die besagen: Grundsätzlich ist jedes Kriterium der Gerechtigkeit, das sich ausschließlich auf die Betrachtung von Verteilungen beschränkt - Herr Henke hat dies, wenn ich mich richtig erinnere, als ergebnisorientierte Handlungsrationalität bezeichnet -, zu kurz gegriffen; denn letztlich kann man die Gerechtigkeit von Allokationen nur dann angemessen beurteilen, wenn man die Entstehung der Allokation mit berücksichtigt. Es gibt radikale Varianten, die besagen: Ausschließlich die Art und Weise des Zustandekommens einer Verteilung ist Maßstab dafür, ob diese Verteilung als gerecht gelten kann oder nicht.

Man kann eine ähnliche Übertragung vornehmen, was Institutionen angeht. Man kann sagen: Institutionen eines bestimmten Typs sind angemessen, sind demokratisch, sind fair. Wenn im Rahmen dieser Institutionen Verteilungen zustande kommen, sind diese Verteilungen gerecht, ganz unabhängig davon, wie sie im Detail aussehen. Es kann einen breiten Spielraum von möglichen Verteilungen geben, die alle mit diesem institutionellen Gefüge kompatibel sind. Juristen tendieren sehr stark zur Betonung dieses Aspekts von Gerechtigkeit.

Die Attraktivität dieses, was Gerechtigkeitsaspekte angeht, doch sehr asketischen Verfahrens der Verteilung von Organen, wie es bislang etabliert ist, hängt damit zusammen, daß ganz offensichtlich die direkte Anwendung von Kriterien der Verteilungsgerechtigkeit die Gesamtproblematik 
von ethisch angemessenem Verhalten und Handeln nicht adäquat erfaßt. Meine These lautet, daß Kriterien der Verteilungsgerechtigkeit nur sinnvoll angewendet werden können, wenn sie in einen umfassenderen ethischen Kontext eingebettet sind.

$\mathrm{Zu}$ diesem ethischen Kontext gehört erstens, daß es sanktionierte Individualrechte gibt, die das, was man unter Aspekten der Verteilungsgerechtigkeit optimiert, einschränken. Dazu gehören zweitens Kooperationsnormen, die sich nicht aus der individuellen Optimierung ergeben. Und drittens gibt es eine Dimension, die u.a. in Frankfurt besonders betont wird, diejenige der Kommunikation, der Diskurs, die Etablierung von Konsensen. Sie beruht darauf, da $\beta$ die Einzelpersonen nicht optimieren, sondern sich zusammensetzen und prüfen: Was kommt aus diesem Diskurs heraus? Ich halte mich an das, worauf wir uns geeinigt haben. Dazu wurde in dem Beitrag von Herrn Eigler ein ganz konkretes Beispiel genannt.

Die Alltagsinteraktion enthält eine Vielfalt von konstitutiven Regeln, auch informellen Institutionen, die einschränkend wirken, auf welche Optimierungsaspekte auch immer man abhebt. In dem dann noch verbleibenden Rahmen sind Kriterien der Verteilungsgerechtigkeit adäquat anwendbar. Nach meiner Auffassung ist dieser Rahmen aber relativ eng.

Verteilungsgerechtigkeit im Rahmen der Konzeption struktureller Rationalität

Strukturelle Einschränkung von optimierendem

Verhalten ist konstitutiv für gesellschaftliches Handeln

- (Sanktionierte) Individualrechte

(Grundrechtskatalog)

- Kooperationsnormen

- Alltags-Interaktion

- Kommunikation etc.

$\Rightarrow$ Strukturelle Einbindung von

Verteilungsgerechtigkeit notwendig 


\section{Sachverzeichnis}

Abrechnungsmodalitäten 334

Abstoßung, Knochenmarktransplantation 203

Akademie für Ethik in der Medizin XVII

Akademie der Wissenschaften und der Literatur Mainz XVII

Allokation 6 juristische Problematik 288

Allokationsnorm 51

Allokationskriterien 234

Allokationsprobleme XVIII

Allokationspyramide 312

Allokationswirklichkeit 51

Alkoholiker, Lebertransplantation 94

Arztzugang 3

Regulierung 3

Aufklärung 290

Ausgaben, GKV 57

Krankenkassen 324

Autoimmunerkrankungen 131

Lebertransplantation 186

Bagatelleistungen 4

Bayesianismus, ethischer 256

Bedarf 154, 169

Bedarfsanalyse 113

Bedarfsplanung 40,180

Großgeräte 40

Bedürnis 169

Beitragsbemessung 3

Beitragsstabilität 51, 326

Budgetierung 335

Budgetrestriktion 68

Bundesverfassungsgericht 302

Chancengleichheit XX

Charakteristika, Nierentransplantation 84

Clubgedanke 270

Common pool ressource (CPR) 268

CPR 268

Deutsche Stiftung Organtransplantation XVII

Diagnostik, Nierentransplantation 188

Diskursethik 261

Dringlichkeit 333
Knochenmarktransplantation 111

Nierentransplantation 188

Lebertransplantation 87

Effekte, intangible 176

Entscheidungsfindung 10

normative Aspekte 13, 339

Kommissionslösung 339

Entscheidungskriterien 233, 242

Entscheidungsmechanismen 339

Entscheidungsprozesse 21

Ethik XVIII, 250, 338

Eurotransplant $234,288,339$

Finanzierung 159

Finanzierungsprinzip 159

Forschungsfragen 340

Ganzkörperbestrahlung 200

Gentechnologie 333

Gerechtigkeit 251

Gerechtigkeitskriterien 262, 281,316

Perspektiven, ordoliberale 262

Gesundheit 22

Ausgaben 30

Bedarf 23

Definition 22

Determinanten 25

Struktur 27

Gesetzliche Krankenversicherung

(GKV) 57

gesetzliche Verankerung 57

Gesundheitsberichterstattung 6,123, 322

Gesundheitsleistungen 50

Struktur 60

Ziele 61

Gesundheitsökonomie $158,326,334$

Gesundheitspolitik 316, 319

zukünftige Perspektiven 320

Gesundheitsversorgung 52

Gesundheitswesen 21, 168

Gleichheit 273

Graft versus Host Reaktion 203

Großgeräte 40

Grundgesetz (GG) 303

Güterabwägung 309

Gutachtertätigkeit 299 
Lebertransplantation $\quad 82$

Selbstbeteiligung 3

SG V 306

Solidargemeinschaft 330

Solidaritätsprinzip 321

Solidarprinzip 333

Spenderentgelt 162

Spenderorganmangel 4

Spenderverfügbarkeit 153

Standard, tradierter 293,311

Standardverfahren 340

Steuerungsmechanismen 36

Strafrecht 304

Struktur, Gesundheitswesen 27

Suppes Gerechtigkeitskriterien 253

Todesfeststellung XVII

Toxische Leberschäden 131

Lebertransplantation 82

Transplantatfunktion, Knochenmark 203

Abstoßung 203

Leukämierezidiv 203

T-Zellen 204

Transplantationsgesetz $\quad \mathrm{X}, 322$

Widerspruchslösung 322

Transplantationsmedizin XVIII, 262

Triage 298

Verfassungsrecht 302

Verrechtlichung 290

Aufklärung 290

Haftpflichtrecht 290

Kostendämpfung 297

Kostendruck 290

Rationalität 292

Sanktionen 292

Standard 293

Standards 290

Verhältnismäßigkeit 291

Warteliste 297

Wirtschaftlichkeitsgebot 295

Versorgung 52

ambulante 30

bedarfsgerechte 52

medizinische 158

stationäre 30

Bedarfsplanung 160

Benutzerkosten 160

Finanzierung, Prinzip, duales 159

Kosten 158

Leistungen 158

Ökonomische Grundlagen 158

Pflegesatz 162

Struktur 158
Bayerische

Staatsbibliothek München
Verteilung, neidfreie 254

Verteilungsentscheidungen 319

Verteilungsgerechtigkeit XX, 250, 286, 302

Bayesianismus, ethischer 256

Bundesverfassungsgericht 302

Eurotransplant 288

Fairness 253

globale

Grundgesetz (GG) 303

Haftungsrecht 288

Handlungsutilitarismus 255

Kriterien 288

Menschenwürde 303

Normen 288

philosophische Sicht 250

Rationalität, utilitaristischkonsequentialistische 250

Sozialhilfe 303

Sozialstaat

Sozialstaatsprinzip 303

Sozialversicherung 303

Verfassungsrecht 302

Verteilungsprobleme 251

Kriterien 251

Pareto-Vergleichbarkeit 252

Praeferenzen 253

Viruserkrankungen, Lebertransplantation 131

Voraussetzungen 186

Knochenmarktransplantation 97

Lebertransplantation 82

Nierentransplantation 82

Warteliste 333

Knochenmarktransplantation 212

Nierentransplantation 236

Lebertransplantation 245

Widerspruchslösung,

Transplantationsgesetz 322

Wirtschaftlichkeit 54

Wirtschaftlichkeitsreserven 326

Wirtschaftlichkeitsuntersuchungen 172

Zuteilung 271

Zuteilungskriterien 234

Gleichheit 273

Lebenschancen 273

Zufallszuteilung 333

Zuteilungswege 245

ärztliche Kriterien 265

Regelung, institutionalisierte 265

Zytostatika 200 